\title{
ADNP, a Microtubule Interacting Protein, Provides Neuroprotection Through End Binding Proteins and Tau: An Amplifier Effect
}

\author{
Illana Gozes ${ }^{1 *}$, Yanina Ivashko-Pachima ${ }^{1}$ and Carmen L. Sayas ${ }^{2}$ \\ ${ }^{1}$ The Lily and Avraham Gildor Chair for the Investigation of Growth Factors, Dr. Diana and Zelman Elton (Elbaum) Laboratory \\ for Molecular Neuroendocrinology, Department of Human Molecular Genetics and Biochemistry, Sackler Faculty of Medicine, \\ Sagol School of Neuroscience and Adams Super Center for Brain Studies, Tel Aviv University, Tel Aviv, Israel, ${ }^{2}$ Centre for \\ Biomedical Research of the Canary Islands, Institute for Biomedical Technologies, Universidad de La Laguna, Tenerife, Spain
}

Keywords: microtubules, microtubule-associated proteins, ADNP, tau, microtubule end binding proteins

\section{ACTIVITY-DEPENDENT NEUROPROTECTIVE PROTEIN (ADNP) INTERACTS WITH MICROTUBULES}

Neuronal plasticity, key to brain function in health, is impaired in neurodevelopmental, neuropsychiatric and neurodegenerative diseases. Neuronal plasticity depends on an intact cytoskeletal system. Here, we focus on recently discovered as well as "classical" cytoskeletal proteins including activity-dependent neuroprotective protein (ADNP), Tau and microtubule end binding proteins (EBs), impacting neuroplasticity and neuropathology.

Original structure-function analysis of astrocyte-secreted protein fragments identified femtomolar-acting neuroprotective peptide moieties VLGGGSALLRSIPA (Brenneman and Gozes, 1996), SALLRSIPA (Brenneman et al., 1998) and NAPVSIPQ (Bassan et al., 1999), with NAPVSIPQ (NAP) being a fragment of ADNP (Bassan et al., 1999). However, ADNP does not only provide neuroprotection through the NAP motif, but is essential for brain formation, with complete Adnp gene knockout in mice resulting in neural tube closure failure and fetal death. Furthermore, NAP promotes neural tube closure in the face of alcohol intoxication (Chen et al., 2005). Thus, the mechanism of ADNP protection, potentially through the potent NAP motif, is of interest.

In search for NAP binding partners, we subjected mouse brain protein extracts to affinity chromatography with NAP as a ligand and identified tubulin as an interacting partner (Divinski et al., 2004, 2006). These results were coupled to NAP promoting changes in microtubule structure and protecting against microtubule disassembly induced by nocodazole in vitro (Gozes and Divinski, 2007) and colchicine in vivo (Jouroukhin et al., 2013). Further data suggested NAP protection against Zinc intoxication, which was originally linked to microtubule disruption (Divinski et al., 2004, 2006) and increased specificity to beta III tubulin, or to neuronal cells (Divinski et al., 2006; Holtser-Cochav et al., 2006). Parallel studies identified reduced axonal transport (Amram et al., 2016), increased tau hyperphosphorylation and tau depositions as a consequence of Adnp haploinsufficiency (Vulih-Shultzman et al., 2007). However, direct interaction of NAP with pure tubulin was not confirmed (Yenjerla et al., 2010). Thus, the discovery of (1) the requirement for the SIP motif on NAPVSIPQ and related peptides for neuroprotection (Wilkemeyer et al., 2003), (2) the SxIP microtubule end binding protein 1 (EB1) interacting motif as a microtubule tip localization signal (Honnappa et al., 2009; Jiang et al., 2012), and (3) EB3 as essential for dendritic spine formation(Jaworski et al., 2009), directed research toward ADNP-NAP$\mathrm{EB} 1 / 3$ interactions. EB1/3 proteins are the master regulators of the microtubule plus-end tracking proteins (+TIPs), which accumulate at the growing ends of microtubules, showing a "comet"

Citation:

Gozes I, Ivashko-Pachima Y and Sayas CL (2018) ADNP, a Microtubule

Interacting Protein, Provides Neuroprotection Through End Binding Proteins and Tau: An Amplifier Effect.

Front. Mol. Neurosci. 11:151 doi: 10.3389/fnmol.2018.00151 pattern at microtubule tips (Lansbergen and Akhmanova, 2006). 


\section{THE IDENTIFICATION OF THE NAP/ADNP EB-DIRECT INTERACTION AND EB REQUIREMENT FOR NAP ACTIVITY}

To establish a direct connection between ADNP and the EB family of proteins, specific immunoprecipitation experiments were carried out, showing direct interactions and enhancement of EB3-ADNP as well as other microtubule plus-end protein interactions by NAP (Oz et al., 2014). Further affinity chromatography with NAPVSIPQ and recombinant EB proteins $(\mathrm{Oz}$ et al., 2014) showed direct interaction with NAP and identified displacement with NAPVSKIPQ (SxIP=SKIP), but not with NAPVAAAAQ. These studies were further elaborated to show direct interactions in COS-7 cells expressing fluorescent EB3 and subject to fluorescent-NAP. Finally, silencing of EB1 and EB3, but not of EB2, abolished NAP protection in the neuronal model pheochromocytoma (PC12) against Zinc intoxication $(\mathrm{Oz}$ et al., 2014), and NAP increased PSD-95 expression in dendritic spines, which was inhibited by EB3 silencing ( $\mathrm{Oz}$ et al., 2014). Together, these studies implicate ADNP/NAP in synaptic plasticity, involving EB proteins (Jaworski et al., 2009; Oz et al., 2014). While these results explained the previously observed NAP interaction with microtubules, bringing into focus the SIP motif (Gozes et al., 2016; Quraishe et al., 2016) and suggesting an amplifier effect at the microtubule tip, the molecular mechanism of increased Tau hyperphosphorylation, as a consequence of ADNP deficiency and protection by NAP against tauopathy (Vulih-Shultzman et al., 2007; Matsuoka et al., 2008; Shiryaev et al., 2009; Jouroukhin et al., 2013), still required further investigations.

\section{TAU REGULATES THE LOCALIZATION AND FUNCTION OF EB1 AND EB3 IN DEVELOPING NEURONAL CELLS AND ANTAGONIZES EB TRACKING AT MICROTUBULE ENDS THROUGH A PHOSPHORYLATION-DEPENDENT MECHANISM}

Tau and EBs were shown to partially co-localize at extending neurites of N1E-115 neuroblastoma cells and axons of primary hippocampal neurons, confirmed by immunoprecipitation and by tau/EB1 direct in vitro pull-down assays (Sayas et al., 2015). Fluorescence recovery after photobleaching assays performed in neuroblastoma cells corroborated tau modulation of EB3 cellular mobility (Sayas et al., 2015). Another excellent report shows that Tau and EBs form a complex via the Cterminal region of $\mathrm{EBs}$ and the microtubule-binding sites of Tau and further show that these two domains are required for the inhibitory activity of Tau on EB localization to microtubule ends. Additionally, their results show that the phosphomimetic mutation S262E within Tau microtubulebinding sites impairs $\mathrm{EB} / \mathrm{Tau}$ interaction and prevents the inhibitory effect of Tau on EB comets (Ramirez-Rios et al., 2016).
The question then arose if there is an EBs/Tau-ADNP/NAP connection.

\section{ADNP/NAP DRAMATICALLY INCREASE MICROTUBULE END-BINDING PROTEIN-TAU INTERACTION: A NOVEL AVENUE FOR PROTECTION AGAINST TAUOPATHY}

We have recently demonstrated that NAP augmented EB1 and EB3 comet density, amounts, length and speed in the N1E-115 neuroblastoma neuronal model. NAP enhanced EB3 homodimer formation, while decreasing EB1-EB3 heterodimer content and driving EB1- and EB3-Tau interactions (dramatic 20 -fold increases), leading to recruitment of EB1/EB3 and Tau to microtubules under zinc intoxication, which has previously been shown to be linked to Tau hyperphosphorylation (IvashkoPachima et al., 2017). As indicated above, our previous results showed that while NAP protected neuronal-like cells against oxidative stress, it did not protect NIH3T3 fibroblasts (Divinski et al., 2004). Indeed, NAP did not protect NIH3T3 cells against zinc intoxication, unless these cells were transfected with Tau. Interestingly, other microtubule-associated proteins (MAPs) may replace Tau; thus, EB-Tau (MAPs) interaction is identified as a novel target for endogenous ADNP neuroprotection (Ivashko-Pachima et al., 2017). Importantly, as indicated, phosphorylation of S262 impaired EB/Tau interactions and our previous data have directly shown that NAP inhibits tau hyperphosphorylation at the S262 site (Jouroukhin et al., 2013) in multiple tauopathy models (Magen et al., 2014), with this phosphorylation site being linked to impaired axonal transport and neurodegeneration (Iijima-Ando et al., 2012), partially solving the NAP/ADNP protective activity against tauopathy (Figure 1). Future studies encompassing the impact of the NAPEBs-Tau interaction on Tau aggregation (Gozes et al., 2014a,b), mitochondrial function (Esteves et al., 2014) and autophagy (Sragovich et al., 2017) are planned and will contribute to clarify the relevance of this protein complex in neuroprotection in the context of tauopathies and other neurodegenerative diseases.

\section{THE ADNP SYNDROME}

The ADNP syndrome (Helsmoortel et al., 2014), a recently described autism spectrum disorder syndrome driven by heterozygous, mostly protein truncating, de novo mutations in ADNP (Gozes et al., 2015, 2017a,b), is a subject of our future studies. These studies are aimed at connecting protein structure to function, with the human condition being characterized with intellectual disability, global developmental delays (including motor delays) and facial dimorphisms. Interestingly, $\sim 80 \%$ of the ADNP children can be identified by premature deciduous tooth eruption, a unique early diagnostic marker. Teething and bone/brain formation converge on mechanisms linked to ubiquitin impacted by the cytoskeleton, paving the path to future research. From a clinical perspective, Coronis Neurosciences 


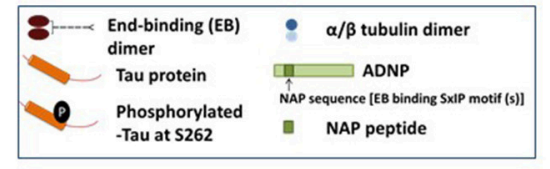

ADNP haploinsufficiency

- Impaired axonal transport

- Neurodegeneration

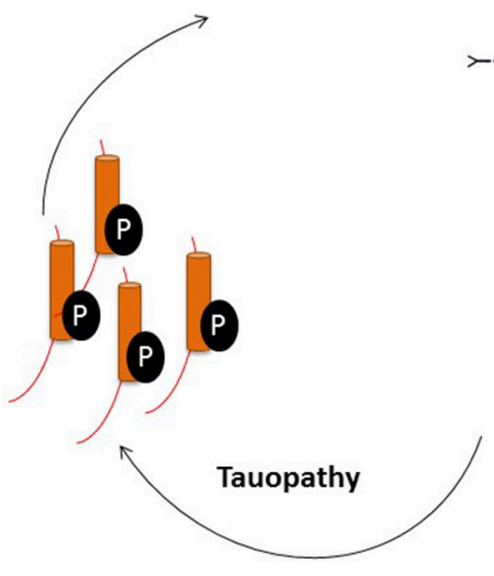

\section{Roles of ADNP - binding to} microtubules

- Axonal transport

- Neuroprotection

Enhancement by NAP transport and synaptic plasticity. ADNP has two adjacent SIP motifs, one within the NAP sequence and one upstream (Bassan et al., 1999; Zamostiano et al., 2001). A NAP_related peptide SKIP (encompassing the SxIP motif), enhances axonal transport in Adnp haploinsufficient mice, probably through the same mechanism (Amram et al., 2016). In tissue culture cells, NAP also enhances dendritic spine formation (synaptic plasticity) through an EB3-depedent mechanism (Oz et al., 2014).

(www.coronisns.com) is developing NAP (CP201) (Magen and Gozes, 2013, 2014) for the ADNP syndrome.

\section{AUTHOR CONTRIBUTIONS}

All authors listed, have made substantial, direct and intellectual contribution to the work, and approved it for publication.

\section{FUNDING}

YI-P was partly supported by a scholarship from the Dr. Miriam and Sheldon G. Adelson Graduate School of the Faculty of Medicine at Tel Aviv University (IG) and this

\section{REFERENCES}

Amram, N., Hacohen-Kleiman, G., Sragovich, S., Malishkevich, A., Katz, J., Touloumi, O., et al. (2016). Sexual divergence in microtubule function: the novel intranasal microtubule targeting SKIP normalizes axonal transport and enhances memory. Mol. Psychiatry 21, 1467-1476. doi: 10.1038/mp.2015.208

Bassan, M., Zamostiano, R., Davidson, A., Pinhasov, A., Giladi, E., Perl, O., et al. (1999). Complete sequence of a novel protein containing a femtomolaractivity-dependent neuroprotective peptide. J. Neurochem. 72, 1283-1293. doi: 10.1046/j.1471-4159.1999.0721283.x paper is part of the graduation requirements. The research was further supported by funds from the Israel Science Foundation (ISF) grant (1424/14), ERA-NET neuron AUTYSM, AMN Foundation, Drs. Ronith and Armand Stemmer and Mr. Arthur Gerbi (French Friends of Tel Aviv University), as well as Canadian (Mrs. Anne and Mr. Alex Cohen) and Spanish Friends of Tel Aviv University. CS research was supported first by CSIC and CIBERNED, in the group of Prof. J. Avila (CBM Severo Ochoa, CSIC-UAM, Madrid), and then as PI by the IMBRAIN project [(FP7-REGPOT-2012-CT2012-31637IMBRAIN), Framework Programme 7 (Capacities)], awarded to the ITB and CIBICAN at ULL. CS is currently supported by ULL and Cabildo de Tenerife, under the Programa Agustín de Betencourt.

Brenneman, D. E., and Gozes, I. (1996). A femtomolar-acting neuroprotective peptide. J. Clin. Invest. 97, 2299-2307. doi: 10.1172/JCI118672

Brenneman, D. E., Hauser, J., Neale, E., Rubinraut, S., Fridkin, M., Davidson, A., et al. (1998). Activity-dependent neurotrophic factor: structure-activity relationships of femtomolar-acting peptides. J. Pharmacol. Exp. Ther. 285, 619-627.

Chen, S. Y., Charness, M. E., Wilkemeyer, M. F., and Sulik, K. K. (2005). Peptide-mediated protection from ethanol-induced neural tube defects. Dev. Neurosci. 27, 13-19. doi: 10.1159/000 084528 
Divinski, I., Holtser-Cochav, M., Vulih-Schultzman, I., Steingart, R. A., and Gozes, I. (2006). Peptide neuroprotection through specific interaction with brain tubulin. J. Neurochem. 98, 973-984. doi: 10.1111/j.1471-4159.2006. 03936.x

Divinski, I., Mittelman, L., and Gozes, I. (2004). A femtomolar acting octapeptide interacts with tubulin and protects astrocytes against zinc intoxication. J. Biol. Chem. 279, 28531-28538. doi: 10.1074/jbc.M403197200

Esteves, A. R., Gozes, I., and Cardoso, S. M. (2014). The rescue of microtubuledependent traffic recovers mitochondrial function in Parkinson's disease. Biochim. Biophys. Acta 1842, 7-21. doi: 10.1016/j.bbadis.2013.10.003

Gozes, I., and Divinski, I. (2007). NAP, a neuroprotective drug candidate in clinical trials, stimulates microtubule assembly in the living cell. Curr. Alzheimer Res. 4, 507-509. doi: 10.2174/156720507783018208

Gozes, I., Helsmoortel, C., Vandeweyer, G., N., Van der Aa, Kooy, F., and Sermone, S. B. (2015). The compassionate side of neuroscience: tony sermone's undiagnosed genetic Journey-ADNP mutation. J. Mol. Neurosci. 56, 751-757. doi: 10.1007/s12031-015-0586-6

Gozes, I., Iram, T., Maryanovsky, E., Arviv, C., Rozenberg, L., Schirer, Y., et al. (2014a). Novel tubulin and tau neuroprotective fragments sharing structural similarities with the drug candidate NAP (Davuentide). J. Alzheimer's Dis. 40(Suppl. 1):S23-S36. doi: 10.3233/JAD-131664

Gozes, I., Patterson, M. C., Van Dijck, A., Kooy, R. F., Peeden, J. N., Eichenberger, J. A., et al. (2017a). The eight and a half year journey of undiagnosed AD: gene sequencing and funding of advanced genetic testing has led to hope and new beginnings. Front. Endocrinol. 8:107. doi: 10.3389/fendo.2017.00107

Gozes, I., Schirer, Y., Idan-Feldman, A., David, M., and Furman-Assaf, S. (2014b). NAP alpha-aminoisobutyric acid (IsoNAP). J. Mol. Neurosci. 52, 1-9. doi: 10.1007/s12031-013-0103-8

Gozes, I., Sragovich, S., Schirer, Y., and Idan-Feldman, A. (2016). D-SAL and NAP: two peptides sharing a SIP domain. J. Mol. Neurosci. 59, 220-231. doi: 10.1007/s12031-015-0701-8

Gozes, I., Van Dijck, A., Hacohen-Kleiman, G., Grigg, I., Karmon, G., Giladi, E., et al. (2017b). Premature primary tooth eruption in cognitive/motor-delayed ADNP-mutated children. Transl. Psychiatry 7:e1043. doi: 10.1038/tp.2017.27

Helsmoortel, C., Vulto-van Silfhout, A. T., Coe, B. P., Vandeweyer, G., Rooms, L., van den Ende, J., et al. (2014). A SWI/SNF-related autism syndrome caused by de novo mutations in ADNP. Nat. Genet. 46, 380-384. doi: 10.1038/ng.2899

Holtser-Cochav, M., Divinski, I., and Gozes, I. (2006). Tubulin is the target binding site for NAP-related peptides: ADNF-9, D-NAP, and D-SAL. J. Mol. Neurosci. 28, 303-307. doi: 10.1385/JMN:28:3:303

Honnappa, S., Gouveia, S. M., Weisbrich, A., Damberger, F. F., Bhavesh, N. S., Jawhari, H., et al. (2009). An EB1-binding motif acts as a microtubule tip localization signal. Cell 138, 366-376. doi: 10.1016/j.cell.2009.04.065

Iijima-Ando, K., Sekiya, M., Maruko-Otake, A., Ohtake, Y., Suzuki, E., Lu, B., et al. (2012). Loss of axonal mitochondria promotes tau-mediated neurodegeneration and Alzheimer's disease-related tau phosphorylation via PAR-1. PLoS Genet. 8:e1002918. doi: 10.1371/journal.pgen.1002918

Ivashko-Pachima, Y., Sayas, C. L., Malishkevich, A., and Gozes, I. (2017). ADNP/NAP dramatically increase microtubule end-binding protein-Tau interaction: a novel avenue for protection against tauopathy. Mol. Psychiatry 22, 1335-1344. doi: 10.1038/mp.2016.255

Jaworski, J., Kapitein, L. C., Gouveia, S. M., Dortland, B. R., Wulf, P. S., Grigoriev, I., et al. (2009). Dynamic microtubules regulate dendritic spine morphology and synaptic plasticity. Neuron 61, 85-100. doi: 10.1016/j.neuron.2008.11.013

Jiang, K., Toedt, G., Montenegro Gouveia, S., Davey, N. E., Hua, S., van der Vaart, B., et al. (2012). A Proteome-wide screen for mammalian SxIP motifcontaining microtubule plus-end tracking proteins. Curr. Biol. 22, 1800-1807. doi: 10.1016/j.cub.2012.07.047

Jouroukhin, Y., Ostritsky, R., Assaf, Y., Pelled, G., Giladi, E., and Gozes, I. (2013). NAP (davunetide) modifies disease progression in a mouse model of severe neurodegeneration: protection against impairments in axonal transport. Neurobiol. Dis. 56, 79-94. doi: 10.1016/j.nbd.2013.04.012

Lansbergen, G., and Akhmanova, A. (2006). Microtubule plus end: a hub of cellular activities. Traffic 7, 499-507. doi: 10.1111/j.1600-0854.2006.00400.x

Magen, I., and Gozes, I. (2013). Microtubule-stabilizing peptides and small molecules protecting axonal transport and brain function: focus on davunetide (NAP). Neuropeptides 47, 489-495. doi: 10.1016/j.npep.2013.10.011
Magen, I., and Gozes, I. (2014). Davunetide: Peptide therapeutic in neurological disorders. Curr. Med. Chem. 21, 2591-2598. doi: $10.2174 / 0929867321666140217124945$

Magen, I., Ostritsky, R., Richter, F., Zhu, C., Fleming, S. M., Lemesre, V., et al. (2014). Intranasal NAP (davunetide) decreases tau hyperphosphorylation and moderately improves behavioral deficits in mice overexpressing alpha-synuclein. Pharmacol. Res. Perspect. 2:e00065. doi: 10.10 $02 / \operatorname{prp} 2.65$

Matsuoka, Y., Jouroukhin, Y., Gray, A. J., Ma, L., Hirata-Fukae, C., Li, H. F., et al. (2008). A neuronal microtubule-interacting agent, NAPVSIPQ, reduces tau pathology and enhances cognitive function in a mouse model of Alzheimer's disease. J. Pharmacol. Exp. Ther. 325, 146-153. doi: 10.1124/jpet.107.13 0526

Oz, S., Kapitansky, O., Ivashco-Pachima, Y., Malishkevich, A., Giladi, E., Skalka, N., et al. (2014). The NAP motif of activity-dependent neuroprotective protein (ADNP) regulates dendritic spines through microtubule end binding proteins. Mol. Psychiatry 19, 1115-1124. doi: 10.1038/mp.20 14.97

Quraishe, S., Sealey, M., Cranfield, L., and Mudher, A. (2016). Microtubule stabilising peptides rescue tau phenotypes in-vivo. Sci. Rep. 6:38224. doi: $10.1038 /$ srep38224

Ramirez-Rios, S., Denarier, E., Prezel, E., Vinit, A., Stoppin-Mellet, V., Devred, F., et al. (2016). Tau antagonizes end-binding protein tracking at microtubule ends through a phosphorylation-dependent mechanism. Mol. Biol. Cell 27, 2924-2934. doi: 10.1091/mbc.E16-01-0029

Sayas, C. L., Tortosa, E., Bollati, F., Ramírez-Ríos, S., Arnal, I., and Avila, J. (2015). Tau regulates the localization and function of End-binding proteins 1 and 3 in developing neuronal cells. J. Neurochem. 133, 653-667. doi: 10.11 $11 /$ jnc. 13091

Shiryaev, N., Jouroukhin, Y., Giladi, E., Polyzoidou, E., Grigoriadis, N. C., Rosenmann, H., et al. (2009). NAP protects memory, increases soluble tau and reduces tau hyperphosphorylation in a tauopathy model. Neurobiol. Dis. 34, 381-388. doi: 10.1016/j.nbd.2009.02.011

Sragovich, S., Merenlender-Wagner, A., and Gozes, I. (2017). ADNP plays a key role in autophagy: from autism to schizophrenia and Alzheimer's disease. Bioessays 39:1700054. doi: 10.1002/bies.201700054

Vulih-Shultzman, I., Pinhasov, A., Mandel, S., Grigoriadis, N., Touloumi, O., Pittel, Z., et al. (2007). Activity-dependent neuroprotective protein snippet NAP reduces tau hyperphosphorylation and enhances learning in a novel transgenic mouse model. J. Pharmacol. Exp. Ther. 323, 438-449. doi: 10.1124/jpet.107.129551

Wilkemeyer, M. F., Chen, S. Y., Menkari, C. E., Brenneman, D. E., Sulik, K. K., and Charness, M. E. (2003). Differential effects of ethanol antagonism and neuroprotection in peptide fragment NAPVSIPQ prevention of ethanolinduced developmental toxicity. Proc. Natl. Acad. Sci. U.S.A. 100, 8543-8548. doi: 10.1073/pnas.1331636100

Yenjerla, M., LaPointe, N. E., Lopus, M., Cox, C., Jordan, M. A., Feinstein, S. C., et al. (2010). The neuroprotective peptide NAP does not directly affect polymerization or dynamics of reconstituted neural microtubules. J. Alzheimer's Dis. 19, 1377-1386. doi: 10.3233/JAD-2010 $-1335$

Zamostiano, R., Pinhasov, A., Gelber, E., Steingart, R. A., Seroussi, E., Giladi, E., et al. (2001). Cloning and characterization of the human activity-dependent neuroprotective protein. J. Biol. Chem. 276, 708-714. doi: $10.1074 /$ jbc.M007416200

Conflict of Interest Statement: The authors declare that the research was conducted in the absence of any commercial or financial relationships that could be construed as a potential conflict of interest.

Copyright (c) 2018 Gozes, Ivashko-Pachima and Sayas. This is an open-access article distributed under the terms of the Creative Commons Attribution License (CC $B Y)$. The use, distribution or reproduction in other forums is permitted, provided the original author(s) and the copyright owner are credited and that the original publication in this journal is cited, in accordance with accepted academic practice. No use, distribution or reproduction is permitted which does not comply with these terms. 